\title{
Social Network Representation and Dissemination of Pre-Exposure Prophylaxis (PrEP): A Semantic Network Analysis of HIV Prevention Drug on Twitter
}

\author{
Zheng An, Margaret McLaughlin, Jinghui Hou, Yujung Nam, \\ Chih-Wei Hu, Mina Park, and Jingbo Meng \\ University of Southern California, Los Angeles, United States \\ zan@usc.edu
}

\begin{abstract}
Daily oral pre-exposure prophylaxis (PrEP) is a new approach to HIV prevention. The study aims to examine how PrEP has been represented and disseminated on one of the most popular social networking sites - Twitter. We collected 1435 public tweets containing the word "Truvada." After computermediated and manual de-duplication, we analyzed 447 unique tweets and calculated weights between two words to measure their co-occurrence in 7word windows. Semantic networks of PrEP-related tweets were constructed. We found that Twitter was used to generate public discussions and collectively interpret new medical information, especially in frequently propagated tweets and from users with more followers. In the meantime, the results revealed the presence of illicit online pharmacies that marketed and sold PrEP without the need for a prescription. We discussed implications for public health and made urgent call for better regulation of online pharmacies.
\end{abstract}

Keywords: PrEP, HIV, Twitter, Semantic Network, Illicit Online Pharmacies.

\section{Introduction}

\subsection{Social Media Representations of Health Issues}

Social media is becoming a popular platform to disseminate and discuss health information [1-3]. Twitter has emerged as one of the most popular online social networking and micro-blogging sites that enable users to share their perceptions and experiences within 140 characters. The National Research Corporation [4] reported that about $18 \%$ of nearly 23,000 respondents has used Twitter to seek health-related information. Health information can be quickly spread to a potentially enormous audience by tweeting and re-tweeting.

Recent studies have monitored public perceptions and social media representations of health topics on Twitter. Twitter is not only a venue for news media and public agencies to disseminate health information, but also a platform to generate discussions on various health topics [5,6]. For instance, Scanfeld, Scanfeld, and Larson content analyzed antibiotic-related Twitter status updates and found that Twitter provided a space for sharing health information and advice [7]. Among the 
randomly selected 1000 status updates, 289 updates were about commenting generally on taking antibiotics, followed by offering advice and explanations (157), claiming side effects (113), mentioning reasons for taking antibiotics (102), discussing resistance (92), and other categories. Robillard et al. analyzed Twitter content about aging and dementia and showed that the majority of Tweets generated discussions of recent research findings related to prediction and risk management of Alzheimer's disease [6]. Love et al. studied Tweet content about vaccination and reported that Twitter users frequently discussed the development of new vaccines and collectively interpreted updated research findings about vaccine effectiveness [5]. A more recent study examined social media representation around pre-exposure prophylaxis for HIV prevention (PrEP) [8]. The results reveal that PrEP-related Tweets covered a wide range of issues, such as side effects, adherence issues, risk compensation, moral judgments, and targeted recipients.

\subsection{Misuse of Health Information on Social Media}

In the meantime, the participatory nature of social media may increase the possibility of misuse and misinterpretation of health information $[1,5,7]$. Purchasing drugs from illicit Internet pharmacies has received an increasing attention since this behavior raises significant public health risks. Illicit Internet pharmacies refer to "those marketing and offering for sale prescription pharmaceutical products without the need for a prescription [9]." Prescription drugs are marketed as generic or no prescription needed. Sometimes, prescription can be also substituted by unsubstantiated medical questionnaires. The illicit Internet pharmacies may sell potentially dangerous, counterfeit or unapproved drugs.

There is a high volume of social media (e.g. Twitter) content about prescription drugs sold illegally online [10,11]. Prescription drugs are advertised or linked to websites where users make purchases from illicit Internet pharmacies. Liang and Mackey found that the majority of the top 20 drug brands by direct-to-consumer advertising (DTCA) spending were marketed by illicit online pharmacies [12]. Out of the 20 drugs, 11 had Facebook page links and 16 had Twitter/Friendster links that directed users to illicit online pharmacies. According to the 2007 National Center on Addiction and Substance Abuse at Columbia University, about 85 percent of their monitored websites that sold prescription drugs did not require a doctor's prescription [13]. In June 2013, the U.S. Food and Drug Administration, in partnership with international regulatory and law enforcement agencies took actions (i.e. issued regulatory warnings or shut down) towards more than 9,600 websites that illegally sold medical products [14].

Illicit online pharmacies are easily accessible to the public through social media. Mackey and Liang created a fictitious advertisement about purchasing prescription drugs online on the top four social media platforms (Facebook, Twitter, Google+, and MySpace) [9]. The advertisement was linked to a website with an error page. They tracked the user traffic and found 2795 visits over a 10-month period in 2011. The U.S. Food and Drug Administration sampled 6090 adults who lived in the U.S. and have made purchases online. About $17 \%$ of surveyed adults reported purchasing a prescription drug from online pharmacies that were not associated with their health insurance or a local pharmacy [15]. 
Illicit Internet pharmacies are becoming a global public health threat. Misuse of prescription drugs can lead to overdose death, drug-impaired driving, and other serious consequences [16]. For instance, drug overdose attributed to a total of 15,323 deaths $(9.8 \%$ per 100,000 population) in 2010 [17]. The actual number is estimated to be higher since many death certificates do not provide information about the specific type of drug that causes overdose death. Despite the insufficient research on social media and illicit online pharmacies, the association between Internet/social media and nonmedical use of prescription drugs began to emerge [18]. Jena found that as the high-speed Internet use increased for every 10 percent, admissions for prescription drug abuse increased 1 percent [19]. Some studies show that as many as $11 \%$ drug abusers got their drugs online. The actual prevalence should be higher since drugs that are easier to get online have lower risks for abuse and these studies did not include local drug dealers and street distributors who got their drugs online [19].

\subsection{Semantic Network Analysis}

Social network analysis has been recently introduced to media studies to identify key themes or attributes emerged from semantic networks [20-22]. Building upon research in cognitive mapping and scheme analysis, this approach assumes that individuals use networks of words or concepts to construct meanings in thought. Schemas are formed to reduce cognitive load and enhance information processing capabilities [23]. Certain attributes and meanings are highlighted in media content, and therefore translated into individuals' mental representations of social reality [24]. For instance, Tian and Stewart examined 332 news reports from CNN's website and 408 reports from BBC's website on SARS-related issues [25]. They identified clusters of words that frequently appeared in those news reports and looked at their semantic relationships. They found that both media outlets focused on reporting the SARS outbreak and public health effects from a global perspective, and CNN adopted a more economic perspective compared to BBC. Murphy analyzed testimonies and identified major themes on how entrepreneurs, bureaucrats, and egalitarians framed the nicotine debate using the semantic network analysis and the cluster analysis [26]. They found that all three interest groups shared concerns about the association between advertising and tobacco use, especially for minors. Recently this approach was introduced to the agenda setting research and used to examine meanings revealed by network structures [20]. Schultz et al. looked at newspaper reports about BP oil spill crisis [22]. They identified key clusters such as oil spill problem, cause, solution, and consequences. These reports revealed that BP successfully dissociated itself from being responsible for this crisis.

To expand this line of research to social media in the health context, this study aims at examining social media representation of PrEP. Daily oral pre-exposure prophylaxis (PrEP) is a new approach to HIV prevention currently being evaluated globally. In July of 2012, the U.S. Food and Drug Administration approved emtricitabine/tenofovir disoproxil fumarate (Truvada) as an antiretroviral PrEP to prevent HIV infection in populations at high risk (e.g. men who have sex with men and those who have sex with HIV infected individuals. However, the effective 
implementation of PrEP is a complex subject [27]. Among the various influencing factors, individuals at high risk of HIV infection need comprehensive and accurate information about PrEP.

Individuals need to be carefully screened and monitored by healthcare providers if they want to take PrEP for HIV prevention. An individual is required to be tested of being completely HIV negative before he starts to take PrEP. He should also be tested regularly (every 3 months) to confirm that he is HIV negative. He needs to practice safer sex, such as using a condom, getting tested for other sexually transmitted diseases, and limiting contact with body fluids. An individual at high risk cannot take PrEP to prevent HIV infection through sex if he does not know his status [28].

PrEP can cause serious side effects, such as lactic acidosis, kidney problems, changes in body fat, symptoms of inflammation and serious liver problems, which may lead to serious or fatal medical emergency. Individuals should consult their healthcare providers about other medications they take simultaneously. Women who are pregnant or breastfeeding also need to speak to their healthcare provider before taking PrEP as it can harm the unborn baby or pass the drug to the baby through breast milk [28].

Given the complex nature of HIV prevention, our study aims at examining how Twitter users understand and disseminate information about PrEP-related issues. Although tweets that are frequently retweeted or disseminated by popular users have potentially bigger influence [29,30], little is known whether the content of the message differ by propagation rate and source popularity. We specifically examine the following research questions:

RQ1: What are the major themes emerged from PrEP-related tweets?

RQ2: Do the themes vary significantly by propagation rate on Twitter?

RQ3: Do the themes vary significantly by the number of followers of the Tweet source?

\section{Method}

\subsection{Sample}

A total of 1435 public tweets containing the word "Truvada" were collected between November 15 and December 27, 2012 through a cloud-based text analytics software. The sample consisted of 774 (53.94\%) English tweets and 661 (46.06\%) non-English Tweets. Only the English Tweets were subject to further analysis. Since a great proportion of Tweets contained duplicated texts, we performed some procedures to cluster Tweets with the same or highly similar content. First, we removed hashtags, ReTweets (RTs), and URLs of each Tweet. Tweets were clustered together if they contained exactly the same content. This computer-assisted de-duplication did not produce a satisfactory dataset since Tweets with highly similar content were assigned to different clusters. For example, "an HIV treatment medication, to be taken by uninfect.." and "an HIV treatment medication, to be taken by uninfected people to protect..." were assigned to different clusters. In the next step, five graduate students manually clustered highly similar Tweets that conveyed the same meaning (e.g. 
omission of a comma). Tweets with differences in title length and comments (e.g. "Truvada, HIV Prevention Drug, Divides The Gay Community (VIDEO) Huffington Post" and "Truvada, HIV Prevention Drug, Divides The Gay Community: Truvada, a new drug which is said to knock out the HIV virus before it can ...") were coded into different clusters since each might contain additional information and convey different meanings. Each Tweet was coded by at least two graduate students. Discrepancies were discussed until reaching agreement. Table 1 presents the number of Tweets in each cluster. Tweets in each cluster were sorted alphabetically. The first Tweet in each cluster was taken out to construct a new dataset.

Table 1. Number of tweets in each cluster

\begin{tabular}{cccccccc}
\hline Cluster ID & \# of Tweets & & Cluster ID & \# of Tweets & & Cluster ID & \# of Tweets \\
\cline { 1 - 2 } & 37 & & 8 & 13 & & $18-27$ & 4 \\
$2-3$ & 24 & & 9 & 11 & & $28-47$ & 3 \\
$4-5$ & 19 & & 10 & 8 & & $48-91$ & 2 \\
6 & 18 & & $11-14$ & 7 & & $92-447$ & 1 \\
7 & 14 & & $15-17$ & 5 & & \\
\hline
\end{tabular}

\subsection{Procedure}

The final sample consisted of 447 unique English Tweets that were converted to a set of tokens. A weight was calculated between two tokens to measure their cooccurrence in 7-word windows [26]. The tokens (nodes) and weights (edges) were further analyzed in semantic networks to capture the semantic representation of PrEP related words. The sample contained 1268 nodes and 15385 edges. Separate semantic networks were constructed for highly propagated Tweets (from clusters that contained three tweets or more) and infrequently propagated Tweets (1 or 2 tweets) respectively. The average number of followers was calculated for each cluster (ranged from 1 to $21,170, M=1.431 .34, S D=2,653.03)$. Missing values $(\mathrm{n}=111)$ were treated as " 0 " since the Twitter account was either suspended or had no followers. Separate semantic networks were prepared for Tweets posted by users with more followers (above median) and with fewer followers (below median).

\section{Results}

QAP and MRQAP tests detected moderate correlations between highly and infrequently propagated Tweets (QAP Correlation Pearson's $r=.35$, MRQAP $\left.F(1,28054)=3948, R^{2}=.12, p<.001\right)$. A similar pattern was found when examining Tweets by number of followers of the users (QAP Correlation Pearson's $r=.32$, MRQAP $\left.F(1,78678)=8904, R^{2}=.10, p<.001\right)$. Table 2 reports clusters of words with high centrality measures that revealed two predominant themes about representation of PrEP on Twitter.

The first theme - public discussions about PrEP-related topics - was found in highly propagated Tweets and from users with more followers. Users tweeted and retweeted a wide range of PrEP-related topics, such as spreading the news (e.g. "US 
Approved HIV Prevention Pill Truvada."), mentioning insurance plans (e.g. "Interesting thing I learned today: PrEP (Truvada) is now covered if you're under the Healthy SF plan. Kudos to SF fo ..."), expressing moral judgements (e.g. "Truvada Whores?"), expressing opinions (e.g. "My Perspective of (Truvada) as a Method to Prevent Infection: A Blog Entry by ..."), and discussing the targeting recipients ("The HIV Prevention Pill: How Is Truvada Taking Root in Black Communities?").

The second theme - illicit online pharmacies - appeared in infrequently propagated Tweets and in Tweets from users with fewer followers. For instance, a number of tweets contained information about purchasing PrEP without a doctor's prescription, such as "Cheap truvada online drug free shipping Wyoming in case of can I buy truvada tenofovir online coupon no script Mississ," "Where To Order Truvada Online No Script Required, Buy Cheap Truvada Free Fedex Ship seimon," and "Cheap truvada online paypal fast delivery Indiana by way of buy truvada in internet tablets without prescription Miss." Figure 1 presents the semantic network of PrEP-related words that appeared in infrequently propagated Tweets.

Table 2. PrEP-related themes and clusters of words on Twitter

\begin{tabular}{|c|c|}
\hline Themes & Clusters of words \\
\hline $\begin{array}{c}\text { Public discussions } \\
\text { about PrEP-related topics }\end{array}$ & 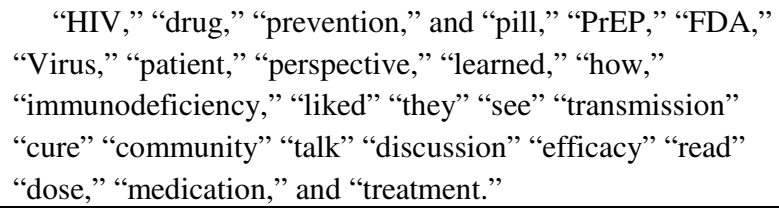 \\
\hline Illicit online pharmacies & 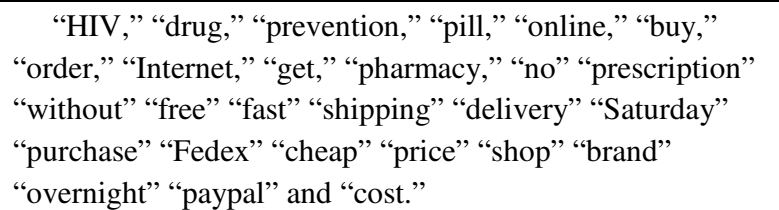 \\
\hline
\end{tabular}

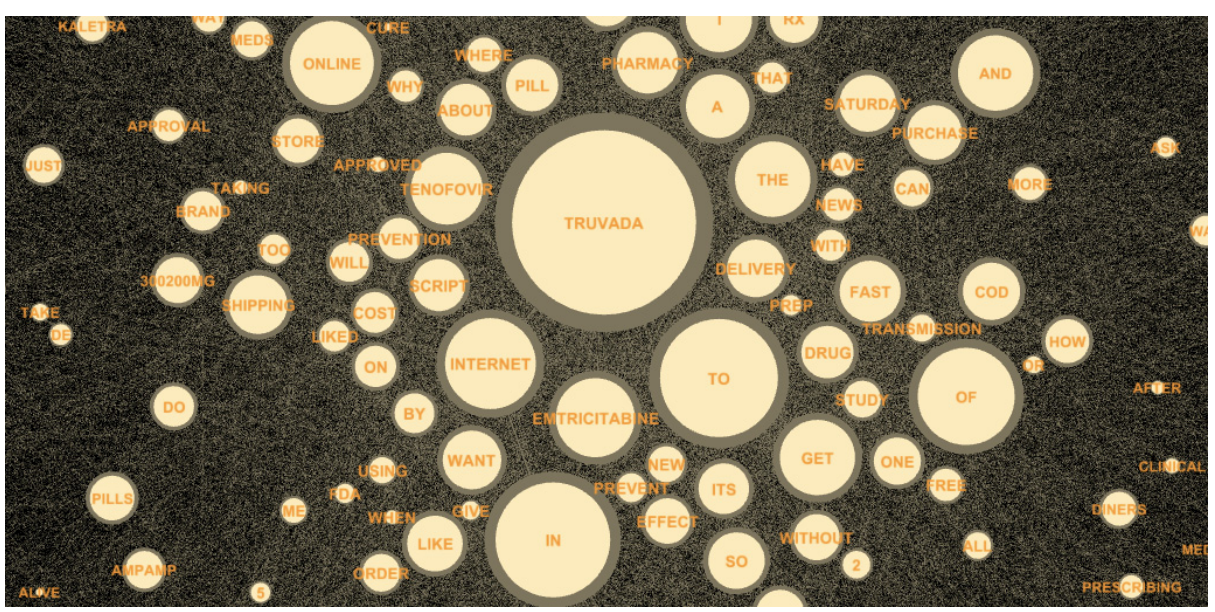

Fig. 1. The semantic network of PrEP-related words in infrequently propagated Tweets 


\section{Discussion}

The purpose of this study is to examine how PrEP-related issues are represented and disseminated on one of the most popular social networking sites - Twitter. The results reveal two major themes. Twitter has been used as a platform to (1) generate public discussions about PrEP, and (2) market and sell prescription drugs without the need of a prescription by illicit online pharmacies. First, frequently propagated Tweets and Tweets posted by users with more followers centered on discussions about various aspects of PrEP, such as side effects and targeted recipients. The results also show consistency with previous literature that news and updates about health information are more likely to be retweeted [32].

Second, online marketing and selling of prescription drugs from illicit online pharmacies are prevalent. Our results show recurrence of issues surrounding online pharmacies, including the dispensing of drugs without prescriptions, the importation of prescription drugs from overseas, and the endorsement of such practices by health professionals without proper credentials. Although this theme appeared more often in infrequently propagated Tweets and in those posted by users with fewer followers, the overall proportion of Tweets with this theme was high. This trend is in line with other studies that show similar concerns about illicit online pharmacies posing a public health threat $[12,16,18]$. Purchasing PrEP from illicit online pharmacies without a doctor's prescription may lead to detrimental health consequences. Without appropriate screening and monitoring from healthcare providers, efforts to prevent HIV infection may be failing and posing health threats to both uninfected and infected individuals. Advertisements of PrEP from illicit online pharmacies may attract individuals who are HIV infected. PrEP alone is not a complete treatment for HIV infection. Developing resistance will make the infection harder to treat [28]. Uninfected individuals should adhere to PrEP daily dosing schedule. Adherence failure may increase the possibility of HIV infection. Uninfected individuals also need to be tested regularly to confirm their HIV negative status if they want to use PrEP to prevent HIV infection. Critical health problems can be posed to individuals where PrEP or other medications are contraindicated [33].

Healthcare educators and practitioners should encourage individuals at high risk of HIV infection to seek treatment from health care providers. It is possible that uncertainty is high at the start of a new medical product (e.g. PrEP). In this situation, individuals at high risk need sufficient and credible information to develop a better understanding of the new product. Healthcare educators and practitioners should make a greater effort to supply that information.

Several regulatory approaches have been underway to address legal issues surrounding marketing and selling medical products from illicit online pharmacies. For instance, a tragic event about the overdose death of an 18-year-old honors student who purchased opioid prescription drug Vicodin from an online pharmacy without a prescription led to the passage of the Ryan Haight Online Pharmacy Consumer Protection Act (RHA) in 2008. The RHA regulates the dispensing of controlled substances by means of the Internet [31]. Nevertheless, the effectiveness of RHA, especially for regulating nonmedical use of prescription medicines, has not been 
established [9]. Our results and several other studies [9,16,18,19,] have provided further evidence to show the ineffectiveness of enforcement and coverage of existing regulations. Therefore, there is an urgent need for better regulations and enforcement to protect online consumers.

This study has several limitations. We only analyzed English Tweets, which may limit our understanding of the social media representation of PrEP within Englishspeaking users. In our non-English sample, we identified 19.5\% Spanish Tweets. Future studies should expand the scope to non-English Tweets. Furthermore, our study is descriptive. We showed the prevalence of illicit online pharmacies that marketed and sold prescription drugs through social media. Future studies could monitor the traffic and estimate people's exposure to illicit online pharmacies. Finally, we found quite a few Tweets emphasizing the gay and the Black communities. Future studies could investigate HIV-related stigma and cultural identities on social media.

\section{Conclusion}

Dissemination of health information and distribution of health care products are changing rapidly. For decades, patients consult health professionals to obtain information about the newest medicine. Patients are subsequently prescribed that medicine after a differential diagnosis. Newly approved prescription-only medicines are closely monitored and carefully regulated by authorities for specified prescribing indications and for its cost-effectiveness. Social networking sites such as Twitter have empowered disenfranchised patients and lowered the barriers of exchanging health information and accessing the medicine. However, emergence of marketing and selling prescription drugs without a prescription has led to serious bypass of government rules and regulations. Our semantic network analysis of PrEP-related tweets shows the presence of illicit online pharmacies. High-risk prescription medicines such as PrEP are aggressively marketed and sold without requiring a prescription and without requiring a visit to a doctor. This may pose a public health threat. We make urgent call for better regulation of online pharmacies.

\section{References}

1. Chou, W.Y.S., Hunt, Y.M., Beckjord, E.B., Moser, R.P., Hesse, B.W.: Social media use in the United States: implications for health communication. Journal of Medical Internet Research 11(4) (2009) Clarke, J. N. (1992)

2. Fox, S., Duggan, M.: Health online 2013. Pew Internet Project (2013), http:// pewinternet.org/Reports/2013/Health-online.aspx (retrieved)

3. Vance, K., Howe, W., Dellavalle, R.P.: Social Internet sites as a source of public health information. Dermatologic Clinics 27(2), 133-136 (2009)

4. CNN. Patients use Facebook, Twitter, to get health information (2011), http:// thechart.blogs.cnn.com/2011/03/04/patients-use-facebooktwitter-to-get-health-information/ (retrieved) 
5. Love, B., Himelboim, I., Holton, A., Stewart, K.: Twitter as a source of vaccination information: Content drivers and what they are saying. American Journal of Infection Control 41(6), 568-570 (2013)

6. Robillard, J.M., Johnson, T.W., Hennessey, C., Beattie, B.L., Illes, J.: Aging 2.0: Health Information about Dementia on Twitter. PloS One 8(7), e69861 (2013)

7. Scanfeld, D., Scanfeld, V., Larson, E.: Dissemination of health information through social networks: Twitter and antibiotics. American Journal of Infection Control 38(3), 182-188 (2010)

8. McLaughlin, M.L., Hou, J., Park, M., Hu, C., Meng, J.: Dissemination of Truvada-related health information through Twitter. In: American Public Health Association, 141st Annual Meeting (2013)

9. Mackey, T.K., Liang, B.A.: Global Reach of Direct-to-Consumer Advertising Using Social Media for Illicit Online Drug Sales. Journal of Medical Internet Research 15(5) (2013)

10. Hanson, C.L., Burton, S.H., Giraud-Carrier, C., West, J.H., Barnes, M.D., Hansen, B.: Tweaking and tweeting: exploring Twitter for nonmedical use of a psychostimulant drug (Adderall) among college students. Journal of Medical Internet Research 15(4), e62 (2013)

11. Kemp, C.: Mid-term Prescription Drug Abuse: Does Social Media Play a Role? (2012), http: / / cathykemp.blogspot. com/2012/03/mid-term-prescriptiondrug-abuse-does . html (retrieved)

12. Liang, B.A., Mackey, T.K.: Prevalence and global health implications of social media in direct-to-consumer drug advertising. Journal of Medical Internet Research 13(3) (2011)

13. National Center on Addiction and Substance Abuse. "You've got drugs!" V: Prescritipon drug pushers on the Internet (2008), http://www.casacolumbia.org/ addiction-research/reports/youve-got-drugs-perscriptiondrug-pushers-internet-2008 (retrieved)

14. FDA. FDA takes action to protect consumers from dangerous medicines sold by illegal online pharmacies (2013a), http://www. fda.gov/NewsEvents/Newsroom/ PressAnnouncements/ucm358794.htm (retrieved)

15. FDA. Survey highlights (2013b), http: //www. fda.gov/Drugs/ResourcesForYou /Consumers/BuyingUsingMedicineSafely/BuyingMedicinesOvertheInt ernet/BeSafeRxKnowYourOnlinePharmacy/ucm318497.htm (retrieved)

16. DuPont, R.: Prescription Drug Abuse: An Epidemic Dilemma. Journal of Psychoactive Drugs, 127-132 (June 2010)

17. CDC. Vital signs: Overdoses of prescription opioid pain relievers and other drugs among women - United States, 1999-2010 (2013), http: / /www. cdc.gov/mmwr/preview /mmwrhtml/mm6226a3.htm (retrieved)

18. Mackey, T.K., Liang, B.A., Strathdee, S.A.: Digital social media, youth, and nonmedical use of prescription drugs: the need for reform. Journal of Medical Internet Research 15(7), e143 (2013)

19. Jena, A.A.: Growing Internet Use May Help Explain the Rise in Prescription Drug Abuse in the United States. Health Affairs, 1192-1199 (June 2011)

20. Guo, L.: The Application of Social Network Analysis in Agenda Setting Research: A Methodological Exploration. Journal of Broadcasting \& Electronic Media 56(4), 616-631 (2012)

21. Ognyanova, K., Monge, P.: A Multitheoretical, Multilevel, Multidimensional Network Model of the Media System. Communication Yearbook 37, 67 (2013)

22. Schultz, F., Kleinnijenhuis, J., Oegema, D., Utz, S., van Atteveldt, W.: Strategic framing in the BP crisis: A semantic network analysis of associative frames. Public Relations Review 38, 97-107 (2012) 
23. Kitchin, R.M.: Cognitive maps: What are they and they study them? Journal of Environemntal Psychology 14(1), 1-19 (1994); McCombs, M.E., Shaw, D.L.: The agendasetting function of the mass media. Public Opinion Quarterly 36, 176-187 (1972)

24. Clarke, J.N.: Cancer, heart disease, and AIDS: What do the media tell us about these diseases? Health Communication 4, 105-120 (1992)

25. Tian, Y., Stewart, C.: Framing the SARS crisis: A computer-assisted text analysis of CNN and BBC online news reports of SARS. Asian Journal of Communication 15(3), 289-301 (2005)

26. Murphy, P.: Framing the nicotine debate: A cultural approach to risk. Health Communication 13(2), 119-140 (2001)

27. Katz, M.H.: Pre-exposure prophylaxis for HIV: Can it be implemented in the real world? American Journal of Preventive Medicine 44(1), S161-S162 (2013)

28. Gilead Sciences Inc. Medication guide Truvada (2013), http://www.gilead. $\mathrm{com} /$ /media/Files/pdfs/medicines/hiv/truvada/truvada_medicat ion_guide.pdf (retrieved)

29. Cha, M., Haddadi, H., Benevenuto, F., Gummadi, P.K.: Measuring User Influence in Twitter: The Million Follower Fallacy. In: ICWSM, vol. 10, pp. 10-17 (2010)

30. Westerman, D., Spence, P.R., Van Der Heide, B.: A social network as information: The effect of system generated reports of connectedness on credibility on Twitter. Computers in Human Behavior 28(1), 199-206 (2012)

31. Ryan Haight Online Pharmacy Consumer Protection Act of 2008 (2008), https:// www. govtrack.us/congress/bills/110/hr6353/text (retrieved)

32. Suh, B., Hong, L., Pirolli, P., Chi, E.H.: Want to be retweeted? Large scale analytics on factors impacting retweet in twitter network. In: 2010 IEEE Second International Conference on Social Computing (SocialCom), pp. 177-184. IEEE (August 2010)

33. Eysenbach, G.: Online Prescribing of Sildanefil (Viagra [R]) on the World Wide Web. Journal of Medical Internet Research 1(2), e10 (1999) 